\title{
Upstream Dosimetry using a Monolithic Active Pixel Sensor (MAPS)
}

\section{R. F. Page ${ }^{* \dagger}$, J. J. Velthuis}

University of Bristol

E-mail: ryan.page@bristol.ac.uk

\section{R. P. Hugtenburg}

Swansea University

\section{S. W. Blake, D. Crawford, S. Fletcher, M. Saunders, P. H. Stevens}

University Hospitals Bristol

\begin{abstract}
Upstream dosimetry has the potential to allow real-time verification and monitoring of Intensity Modulated Radiotherapy (IMRT). This is becoming increasingly important as treatments become ever more complex. The prototype presented here, based on a Monolithic Active Pixel (MAPS) sensor, achieves both precise and accurate dose and Multileaf Collimator (MLC) measurements needed for treatment verification.
\end{abstract}

Technology and Instrumentation in Particle Physics 2014,

2-6 June, 2014

Amsterdam, the Netherlands

\footnotetext{
${ }^{*}$ Speaker.

$\dagger$ Manuscript received July 23, 2014. The research presented here is funded by the National Institute for Health Research (NIHR) Invention for Innovation (i4i)programme. This paper presents independent research commissioned by the National Institute for Health Research (NIHR) under the Invention for Innovation (i4i)programme. The views expressed in this paper are those of the author(s) and not necessarily those of the NHS, the NIHR or the Department of Health.
} 


\begin{tabular}{|l|c|l|}
\hline Parameter & Value & Unit \\
\hline Number of Pixels & $4096 \times 4096$ & Pixels \\
\hline Pixel Pitch & 14 & $\mu \mathrm{m}$ \\
\hline Sensitve Area & $57.3 \times 57.3$ & $\mathrm{~mm}^{2}$ \\
\hline Frame Rate & 40 & frames per second \\
\hline Noise & 83 & e-rms (without CDS) \\
\hline Full Well Capacity & 75,000 & electrons \\
\hline Dynamic Range & 59 & dB (without CDS) \\
\hline
\end{tabular}

Table 1: Table listing some of the key parameters of the Achilles sensor.

\section{Introduction}

Radiotherapy is used to treat cancerous tumours using high energy X-rays, typically $6 \mathrm{MeV}$ or above. In Intensity Modulated Radiotherapy (IMRT) the treatment beam is shaped using Multileaf Collimators (MLC). The MLCs allow complex dose distributions to be created, which has the two-fold advantage of concentrating the dose in the tumour whilst sparing healthy tissue. This is very important, with new IMRT techniques seeking to reduce these margins as far as possible. The importance of IMRT was highlighted by the UK National Cancer Action Team, who are pushing for $30 \%$ of all radiotherapy courses to be carried out using IMRT [1]. The complex nature of IMRT is not without risk. This was highlighted in the report Towards Safer Radiotherapy [2], which recommended independent in-vivo dosimetry. Current methods to perform in-vivo dosimetry include transmission dosimetry (upstream), portal imaging (downstream) and dosimeters placed on the patient [3] [4]. The upstream approach has the benefit of not having scattering coming from the patient, however being upstream requires a very thin detector to minimise attenuation and surface dose [5]. Current devices including the DAVID [6] and COMPASS [7] have attenuation of the order $\sim 5-10 \%$. To minimise this further a Monolithic Active Pixel Sensor (MAPS) was used to build a prototype, this was based on the initial work described here [8], which also resulted in intellectual property being generated [9]. The trend in treatments to be more dynamic also motivates MAPS technology, with good $\mathrm{S} / \mathrm{N}$ and fast frame rates making real-time monitoring and verification a very real possibility [10] [11].

\section{Proof of Concept Device and Experimental Setup}

The proof of concept prototype consists of an Achilles [12] MAPS, a camera system and a DAQ PC. The sensor was mounted on a retrofitted Linac accessory draw. This setup can be seen in figure 1.

The specifications of the Achilles sensor can be seen in table 1. The Achilles was built using $0.35 \mu \mathrm{m}$ CMOS technology with 3T pixels and a $15 \mu \mathrm{m}$ epitaxial layer giving a total thickness of $100 \mu \mathrm{m}$. This equates to an attenuation of $\sim 0.1 \%$ for $2 \mathrm{MeV}$ X-rays [13].

The camera system was operated at $10 \mathrm{fps}$ for the results presented here, but is capable of running up to $40 \mathrm{fps}$. The Linac itself was operated at the nominal 400 Monitor Units/min, which equates to a pulse repetition frequency of $400 \mathrm{~Hz}$. To allow both MLC position and dose reconstruction to be tested runs with Gafchomic RTQA2 film and IBA's MatriXX were taken. These 
deveices were positioned at the iso-centre. A series of exposures were then performed with various MLC configurations and an IMRT anterior head and neck field to allow both MLC position and dose reconstruction to be tested.

\section{Reconstruction Methods and Results}

The reconstruction of the MLC position was performed using the Sobel edge detection kernel. The response of this is gaussian in the present of a sigmoid intensity change. From this a point along the MLC edge can be extracted by fitting a gaussian and evaluating the mean. The final position is found by fitting a linear function to the set of points that make up the MLC edge, a more detailed description can be found here [14]. To check the precision of this method the distribution of 100 single frames were analysed. The result was a precision of $6 \mu \mathrm{m}$ for 10 seconds of data and $52 \pm 4 \mu \mathrm{m}$ for a single frame at the isocentre. The accuracy of the reconstruction was tested by calculating the difference between two parallel MLCs using both the upstream detector and Gafchomic film. The result for the $5 \times 5 \mathrm{~cm}^{2}$ field is shown in figure 2 . The data set was fitted with a linear function and if both sets of data agreee the result of the fit would be a gradient of 1 and an intercept of 0 . The fit result gave a gradient of $1.00 \pm 0.05$ and a intercept of $0.1 \pm 0.5 \mathrm{~mm}$, clearly showing that the upstream reconstruction was accurate to within the precision of the film.

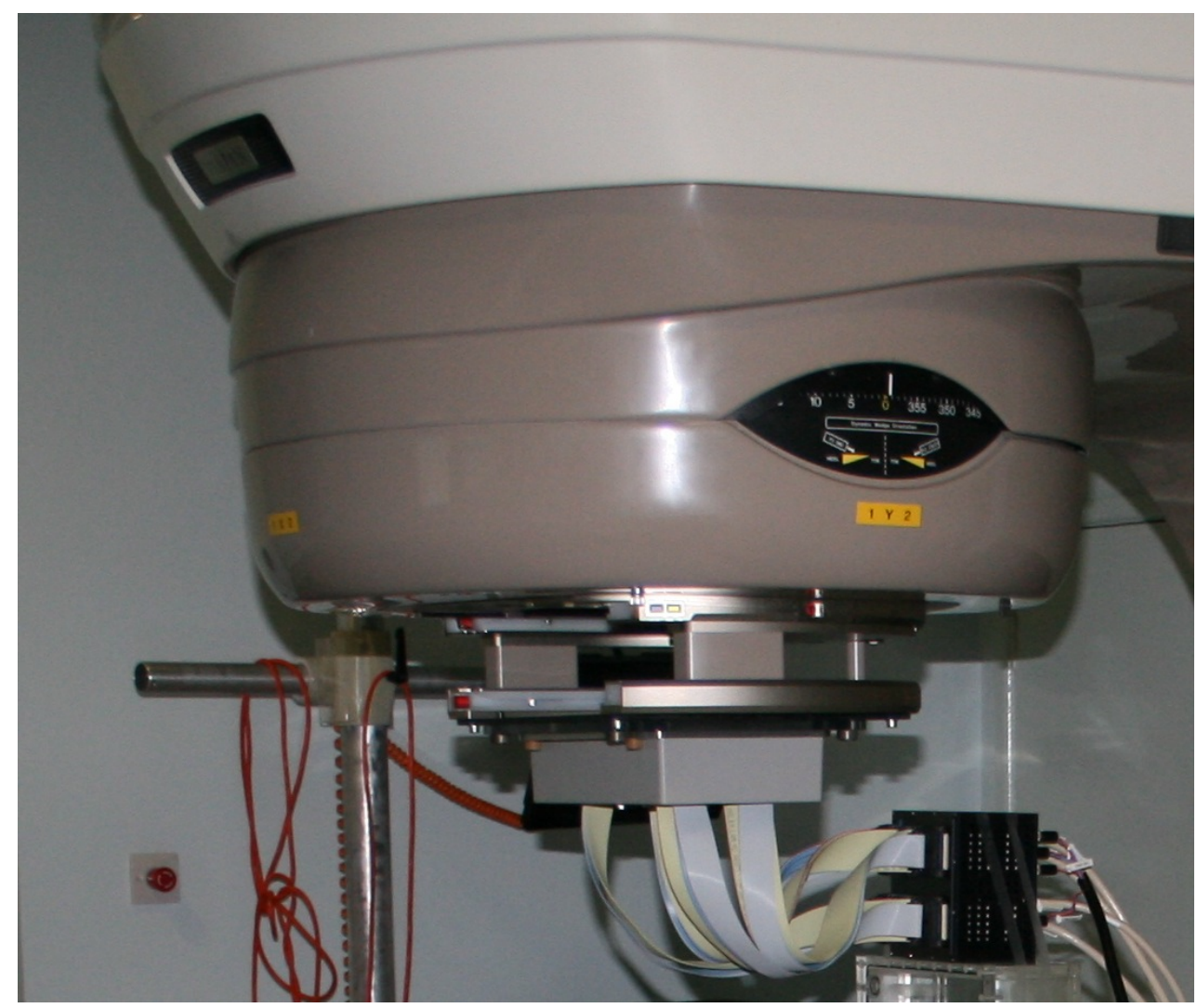

Figure 1: The Achilles sensor fixed to the accessory draw and fixed to the linac head. 


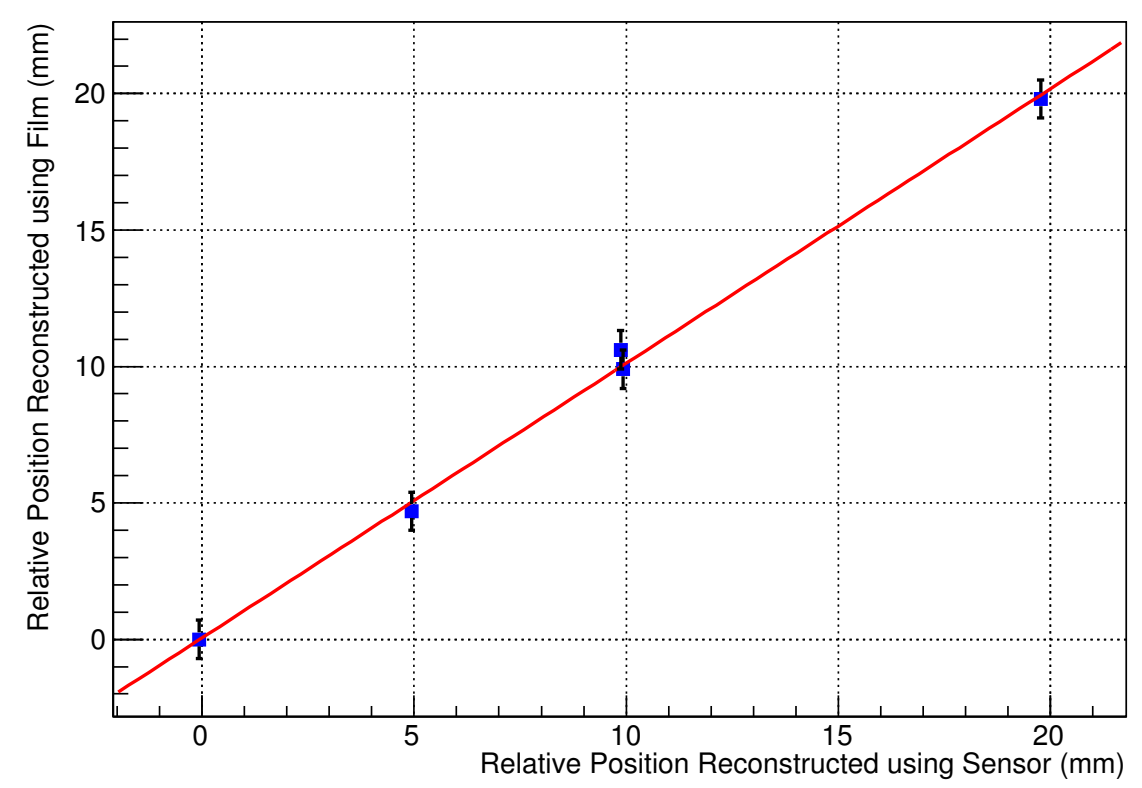

Figure 2: The relative reconstructed position of MLCs for measurements made with the sensor and those made with radiochromic film.

This experiment was repeated with different square fields to ensure that the method was independent of field size. The results of the linear fits are shown in figure 3 and 4 for the gradient and the intercept respectively. From this it can be seen the method does not have a dependance on field size. This is important as IMRT treatments have many field configurations and having a method that removes complex correction factors makes the method very robust.

The dose was reconstructed by first calibrating a MC simulation of the linac and the detector with data from an $8 \times 8 \mathrm{~cm}^{2}$ open field. The photon fluence was then extracted from the data for the IMRT fields by subtracting the MC calculated electron background. The final step was to propagate the photon fluence using a pencil beam kernel to determine the dose at the isocentre where the MartiXX was positioned. The two distributions are shown in figure 5 and were then compared using the gamma metric [15]. The output of which is also in figure 5. For the pass condition of $3 \%$ and $3 \mathrm{~mm} 97 \%$ of the points passed. At this precision the method is good enough for treatment verification.

\section{Conclusion}

The work presented here shows that an IMRT verification and monitoring system based on a MAPS detector is very promising. The MLC positions can be measured to an unprecedented precision and the dose can be calculated with the precision required. The next steps are to improve the DAQ system so that the algorithms developed can be run in realtime. The ultimate goal is to be able to monitor and verify treatments in realtime. This gives the radiographer treatment time feedback allowing for rapid intervention if necessary and reduces the treatment time by removing the pretreatment verification step. 


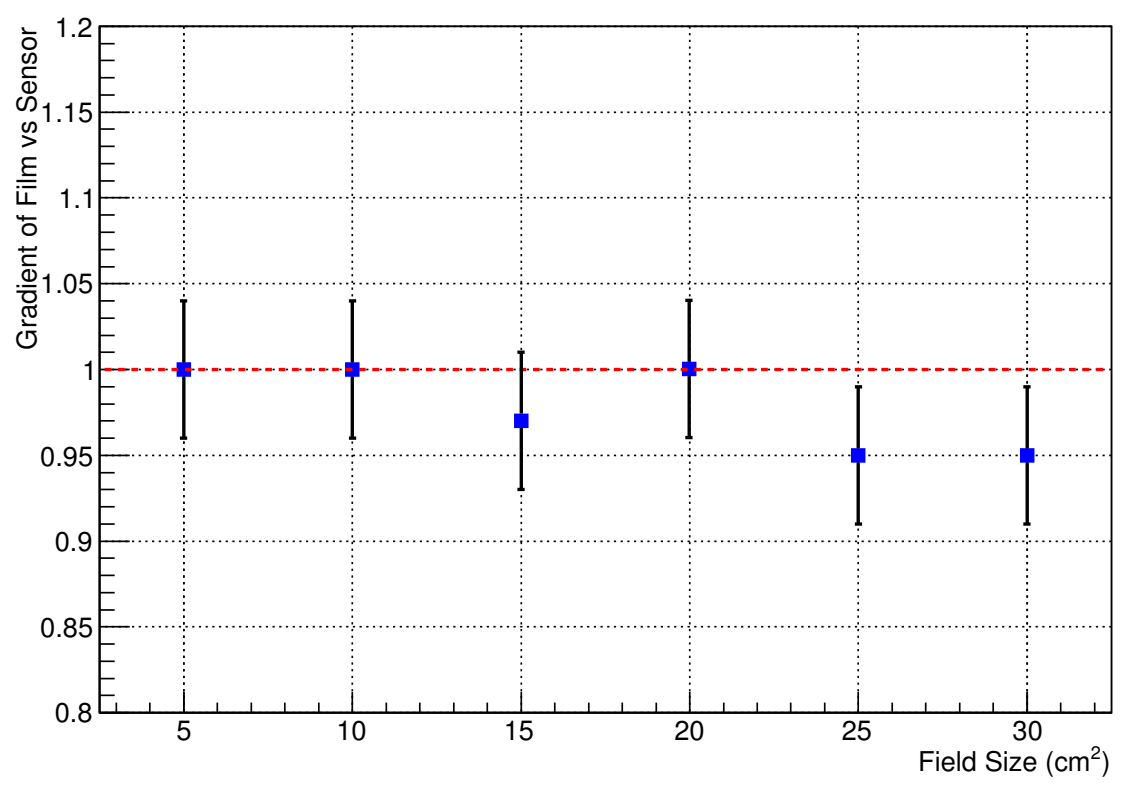

Figure 3: The gradient obtained for a linear fit to the relative reconstructed position measurements for different field sizes.

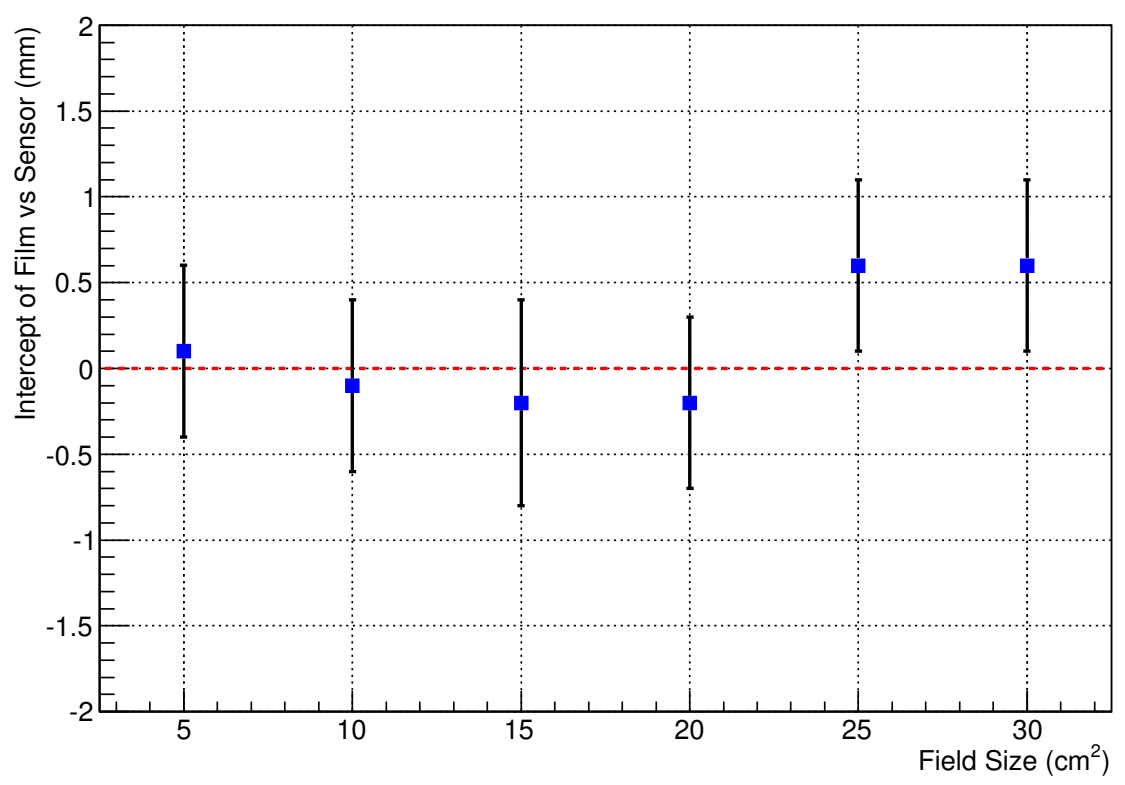

Figure 4: The intercept obtained for a linear fit to the relative reconstructed position measurements for different field sizes. 

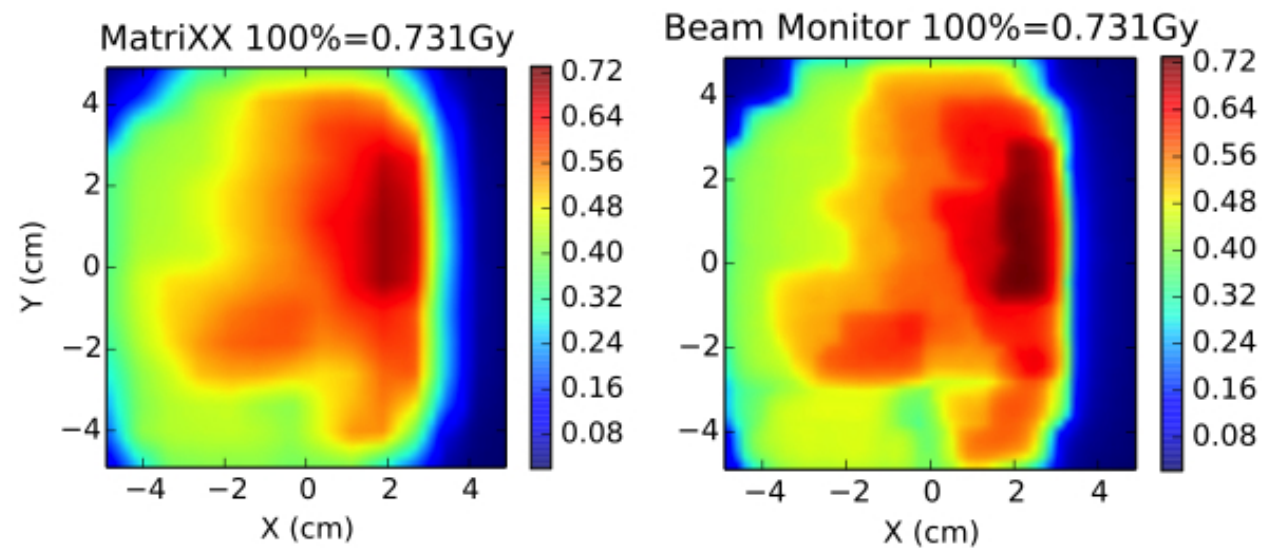

Gamma 3.0\% 3.0mm ref. 0.466GY

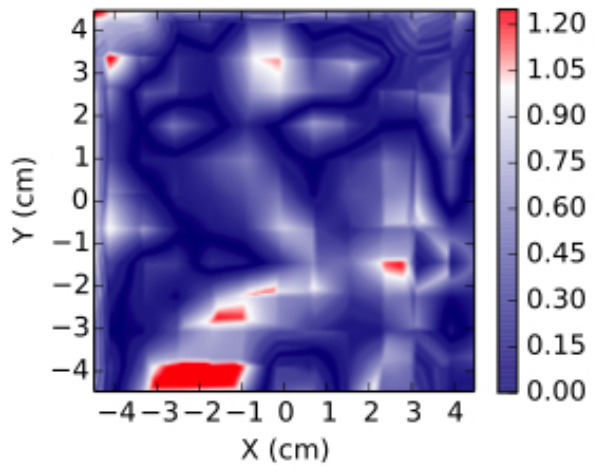

Figure 5: The dose distribution for the upstream detector (top right) and the MatriXX (top left) and the result of the gamma evaluation (bottom).

\section{References}

[1] NHS. UK National Cancer Action Team. Website, 2013.

[2] Towards Safer Radiotherapy. The British Institute of Radiology, The Institute of Physics and Engineering in Medicine, The National Patient Safety Agency, The Society and College of Radiographers and The Royal College of Radiologists, 2008.

[3] D. A. Low, J. M. Moran, J. F. Dempsey, L. Dong, and M. Oldham. Dosimetry tools and techniques for imrt. Med Phys., 38(3):1313-38, Nov 2011.

[4] A. J. Vinall, A. J. Williams, V. E. Currie, A. V. Esch, and D. Huyskens. Practical guidelines for routine intensity-modulated radiotherapy verification: pre-treatment verification with portal dosimetry and treatment verification with in vivo dosimetry. Br J Radiol, 83(995):949-57, Nov 2010.

[5] B. Poppe and et al. Clinical performance of a transmission detector array for the permanent supervision of imrt deliveries. Radiotherapy and oncology, 95(2):158-165, May 2010.

[6] B. Poppe and et al. David-a translucent multi-wire transmission ionization chamber for in vivo verification of imrt and conformal irradiation techniques. Phys. Med. Biol., 51(5):1237-48, 2006.

[7] S. Venkataraman and et al. The influence of a novel transmission detector on $6 \mathrm{mv} x$-ray beam characteristics. Phys. Med. Biol., 54(3173-83), 2009. 
[8] J. Velthuis, R. Hugtenburg, D. Cussans, M. Perry, C. Hall, P. Stevens, H. Lawrence, and A. McKenzie. The VANILLA sensor as a beam monitoring device for x-ray radiation therapy. Applied Radiation and Isotopes, 83, Part $\mathrm{A}(0): 8$ - 11, 2014.

[9] J. Velthuisa, R. Hugtenburg, C. Hall, R. Page, and P. Stevens. Upstream direct xray detection wo2012/085507 a2. International Patent Application, 122011.

[10] H. M. Zin and et al. Towards real-time VMAT verification using a prototype, high-speed cmos active pixel sensor. Phys. Med. Biol., 58(10):3359-75, 2013.

[11] J. Velthuis, R. Page, R. Hugtenburg, S. Blake, D. Crawford, S. Fletcher, M. Saunders, and P. Stevens. An Intensity Modulated Radiotherapy Beam Monitoring System using a Monolithic Active Pixel Sensor. In Radiotherapy and Oncology, volume 110, February 2014.

[12] R. Turchettaa, N. Guerrini, and I. Sedgwick. Large Area CMOS Image Sensors. Journal of Instrumentation, Volume 6, January 2011. Previously presented at IWORID, 11-15th July 2010.

[13] J. H. Hubbell and S. M. Seltzer. Tables of X-ray mass attenuation coefficients and mass energy-absorption coefficients (version 1.4). http://physics.nist.gov/xaamdi (accessed 2012/4/3) National Institute of Standards and Technology, Gaithersburg, MD, 2012.

[14] R. Page, N. Abbott, J. Davies, E. Dyke, H. Randles, J. Velthuis, S. Fletcher, S. Gregory, C. Hall, A.-M. John, H. Lawrence, P. Stevens, R. Hugtenburg, and V. Tunbridge. Using a monolithic active pixel sensor for monitoring multileaf collimator positions in intensity modulated radiotherapy. Nuclear Science, IEEE Transactions on, PP(99):1-5, 2014.

[15] D. A. Low, W. B. Harms, S. Mutic, and J. A. Purdy. A technique for the quantitative evaluation of dose distributions. Medical Physics, 25(5):656-661, 1998. 